\title{
VARIEDADES
}

\section{Novedades sobre la datación de los manuscritos árabes de la Escuela de Estudios Árabes de Granada a partir de su estudio codicológico*.}

\section{New Research on the Dating of the Arabic Manuscripts of the School of Arabic Studies in Granada through Codicology}

\author{
Sonsoles González-García \\ Universidad de Granada \\ orcid.org/0000-0001-7945-6593 \\ Domingo Campillo-García \\ Universidad de Murcia \\ orcid.org/0000-0001-9942-8346 \\ Teresa Espejo-Arias \\ Universidad de Granada \\ orcid.org/0000-0001-8081-559X
}

La datación de los manuscritos es una tarea ardua, en especial cuando carecen de data expresa o-incluso si esta aparece de manera expresa- cuando entran en juego factores como la posibilidad de hallarnos ante copias sucesivas que continúan y repiten modos de ejecución y modelos establecidos.
Dating manuscripts is a difficult task - particularly when these are not expressly datemarked, but even when they are. Factors such as the existence of successive copies that continue and repeat established models and production methods make dating a challenging task. The present work forms part of a much

* Este trabajo se enmarca dentro del proyecto "Nuevas alternativas al conocimiento de los materiales y los procesos de conservación y restauración de obra gráfica y patrimonio documental" (MAT2014-58659-P), financiado por el Ministerio de Economía y Competitividad.

Copyright: (C) 2017 CSIC. Este es un artículo de acceso abierto distribuido bajo los términos de la licencia de uso y distribución Creative Commons Attribution (CC-by) España 3.0. 
Este trabajo se enmarca dentro de una investigación extensa e integral sobre la materialidad de los manuscritos datados y encuadernados de la Escuela de Estudios Árabes (CSIC) de Granada ${ }^{1}$. La aplicación de un protocolo de estudio ${ }^{2}$ ha permitido detectar y corregir errores y proponer una datación alternativa en la datación de los manuscritos ms6, ms10, ms26 y ms29.

Palabras clave: Escuela de Estudios Árabes (Granada); manuscritos árabes; análisis material; datación. broader, comprehensive, investigation on the material composition of dated and bound manuscripts from the School of Arabic StudiesCSIC of Granada. By means of a study protocol, errors in the dating of manuscripts ms6, ms10, ms26 and ms29 are successfully detected and corrected, and alternative dating proposed.

Keywords: School of Arabic Studies; Arabic manuscripts; material analysis; dating.

\section{Introducción}

La datación de los manuscritos árabes es una tarea difícil si tenemos en cuenta las sucesivas copias, la continuidad de los procesos de ejecución y la subsiguiente repetición en los modelos establecidos. Los estudios que tradicionalmente se han llevado a cabo sobre manuscritos árabes se han centrado fundamentalmente en los estudios filológicos y las catalogaciones que de ellos se han realizado recogen, con muchos vacíos, unos pocos aspectos formales que dejan a un lado elementos codicológicos de referencia.

El análisis codicológico que incluye el estudio de los materiales, de las técnicas y de los procesos de encuadernación, permite a los investigadores comprender su manufactura y corregir errores heredados - al considerar aspectos materiales únicamente desde la percepción visual-y la aproximación al marco de ejecución y a la fecha de datación.

El origen de la colección de manuscritos árabes de la Biblioteca de la Escuela de Estudios Árabes de Granada es suficientemente conocido $^{3}$. Ha sido descrita, catalogada y estudiada en distintas ocasiones ${ }^{4}$. En fecha reciente ha sido además digitalizada y puesta a disposición

\footnotetext{
${ }^{1}$ González García, Estudio de las encuadernaciones originales datadas de la Colección de manuscritos árabes de la Biblioteca de la Escuela de Estudios Árabes de Granada.

${ }^{2}$ Espejo y Beny, "Protocolo de descripción del manuscrito andalusí".

3 Álvarez de Morales y Orihuela Uzal, La casa del Chapiz.

${ }^{4}$ Castillo Castillo, "Manuscritos arábigos que se conservan en la Escuela de Estudios Árabes de Granada"; Ávila, Font y de la Torre, Manuscritos árabes y fondo antiguo de la Escuela de Estudios Arabes; González García, Estudio de las encuadernaciones originales datadas.
} 
de la comunidad investigadora a través del proyecto Manuscripta ${ }^{5}$. No obstante, todos los investigadores que se han enfrentado a la colección granadina coinciden en la necesidad de seguir estudiando en profundidad este singular fondo compuesto por 63 volúmenes y 134 obras. Procedentes del fondo antiguo de la Universidad de Granada (mss 1-21), de la familia Granados Montoro (mss 22-44) y de los fondos Carlos Quirós (mss 45-63), la colección contiene principalmente obras de contenido religioso, aunque también incluye obras de derecho, literatura, lexicografía, gramática y poesía que, desde nuestra óptica particular, conforman un corpus excelente para avanzar en el conocimiento de la codicología islámica.

Para el estudio general $l^{6}$, se seleccionaron únicamente los ejemplares datados que conservaban la encuadernación en base a los datos recogidos en el proyecto Manuscripta. De ellos contienen una única obra los ms1, ms2, ms6, ms11, ms18, ms21, ms32 y ms37 —datados según catálogo entre los siglos XII al XIX- y son códices misceláneos ${ }^{7}$, el $\mathrm{ms} 4, \mathrm{~ms} 10, \mathrm{~ms} 19, \mathrm{~ms} 24, \mathrm{~ms} 26, \mathrm{~ms} 29$ y ms 33 - con obras datadas entre los siglos XIV al XIX.

Sobre ellos se ha aplicado un protocolo de caracterización ${ }^{8}$ elaborado ex profeso para el conocimiento de los manuscritos árabes basado en un minucioso estudio codicológico que contempla un completo análisis de los materiales. Los datos obtenidos para cada manuscrito han sido comparados y puestos en relación con el resto de los manuscritos de la colección estudiados así como con los obtenidos de otros ejemplares analizados y de las referencias consultadas.

Hemos resaltado aquellos datos que han resultado determinantes en nuestra investigación. En concreto, los relacionados con la materiali-

${ }^{5}$ El portal Manuscript@CSIC presenta las colecciones de manuscritos en hebreo, árabe, aljamiado, persa y turco conservados en las bibliotecas del CSIC, incorporando tanto su catalogación como su digitalización, en http://manuscripta.bibliotecas.csic.es

${ }^{6}$ González García, Estudio de las encuadernaciones originales datadas.

${ }^{7}$ Es una encuadernación en un volumen de publicaciones bibliográficamente independientes cuya compilación no se justifica por motivos de conservación como sucede en un volumen facticio. Véase "miscelánea" y "facticio" en Martínez de Sousa, Diccionario de bibliología y ciencias afines.

${ }^{8}$ Protocolo de actuación elaborado en el proyecto Caracterización de los materiales de manuscritos árabes de la Península Ibérica para la elaboración de un corpus documental. Proyecto de Investigación de Excelencia, P08-HUM-04188 (2009-2014), Junta de Andalucía, Consejería de Innovación, Ciencia y Empresa, dirigido por Teresa Espejo Arias (Universidad de Granada). 
dad. Como cabía esperar han resultado de especial interés los datos relativos al soporte escritorio (características morfológicas y composición del papel) así como presencia y estudio de filigranas ${ }^{9}$ o la composición de las tintas, entre otros.

Con los resultados obtenidos se concluyó que al menos las datas expresadas en los catálogos para los mss 6, 10, 26 y 29 no se corresponden con el periodo de ejecución que se desprende del citado análisis material.

Figura 1

Manuscritos seleccionados
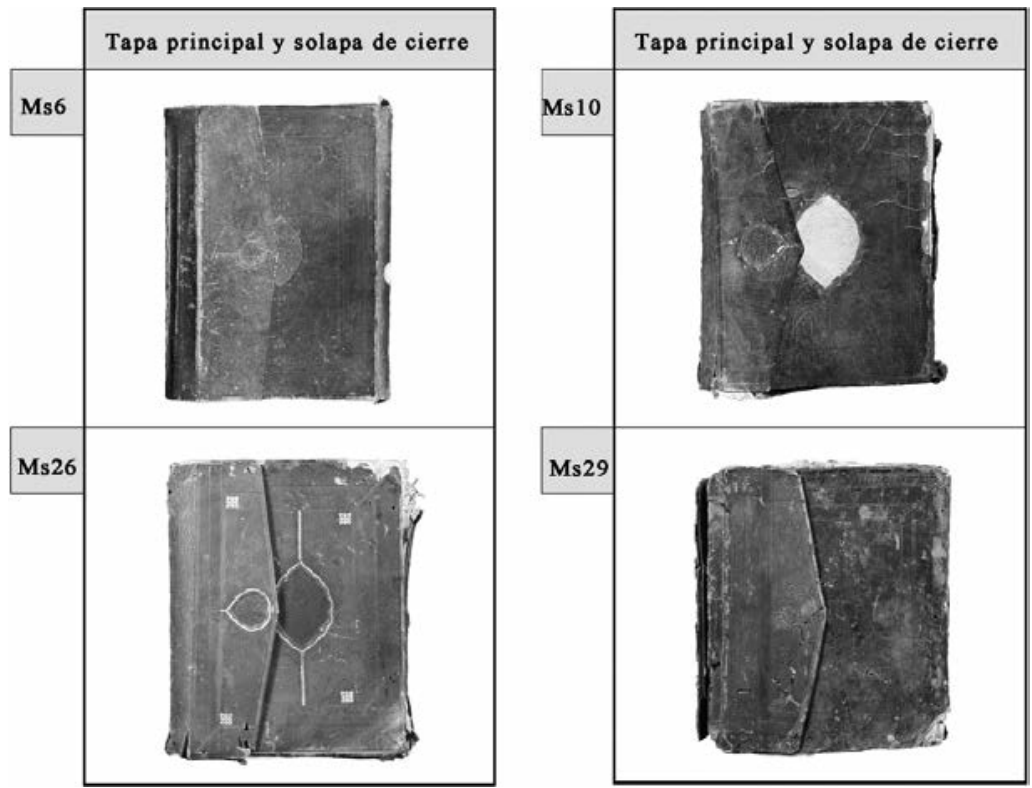

A continuación pasamos a desarrollar los aspectos citados.

\footnotetext{
${ }^{9}$ Para una aproximación en la localización de las diferentes marcas de agua y su relación con la data de los documentos objeto de estudio se han cotejado los diferentes motivos con referencias incluidas en diferentes catálogos, como el de Basanta Campos, Marcas de agua en documentos de los archivos de Galicia, o las bases de datos online del proyecto Bernstein: http://www.memoryofpaper.eu, donde se encuentran las bases de filigranas de Piccard, Briquet, WILC, WZMA, WZIS y WIES.
} 


\section{Nuevas aportaciones para la datación de los documentos de la Colección}

\section{El manuscrito ms6}

El ms6 es una obra de carácter religioso copiada, según las catalogaciones existentes, en el año 1190 d.C. Se trata de Qișaș al-Qur'ān: bad' al-jalq wa-qișaș al-anbiyā' de al-Hayṣam b. Muḥammad alBušanŷ̀1.

El cuerpo del libro combina tres tipos de papel. El primero se corresponde con las guardas y charnelas, es muy blanco y de fabricación industrial y se relaciona con reparaciones o ajustes de la encuadernación. El segundo está formado por un bifolio verjurado que conforma el inicio del volumen y en el que se inicia el texto - $\mathrm{f}$. $2 \mathrm{v}-\mathrm{y}$ un folio en blanco, también verjurado, que cierra el cuerpo del libro. Ambos están filigranados aunque los diseños de filigrana son diferentes - un motivo inclasificable en el bifolio de inicio y un motivo de racimo de uvas en el folio final cuyo ámbito de desarrollo se inicia entre los siglos XV y XVIII- (figura 2). El tercero ocupa el resto del volumen y sus características lo ponen en relación directa con los procedimientos de ejecución del papel árabe. No presenta marcas distintivas, su grado de refino es bajo, resulta difícil observar los signos de la forma empleada para su elaboración y está ligeramente satinado con marcas evidentes del instrumento de lisura.

Figura 2

Filigranas ms6

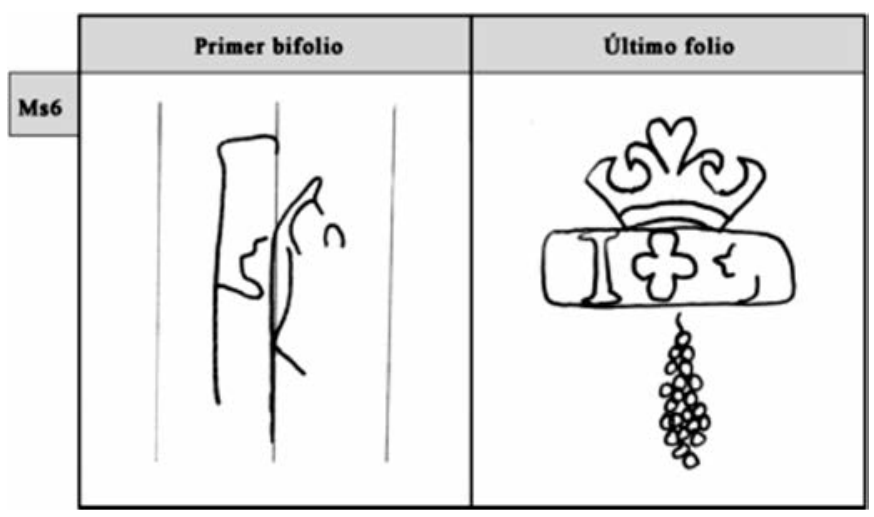

Al-Qantara XXXVIII 1, 2017, pp. 99-111 ISSN 0211-3589 doi: 10.3989/alqantara.2017.004 
Aunque desconocemos el motivo de la combinación y disposición de ambos tipos de papel, se descarta el hecho de que el segundo fuera un añadido posterior pues el texto, realizado aparentemente por una misma mano, se inicia en la última página del primer cuaderno - $\mathrm{f}$. $2 \mathrm{v}-\mathrm{y}$ continúa hasta el final de la obra. Si a esto añadimos que la foliación parece corresponderse con la ejecución del texto y abarca los tres tipos de papel, incluidas las guardas, podríamos pensar incluso que los tres papeles se utilizaron simultáneamente para la copia del texto en una misma secuencia temporal.

De acuerdo con lo anterior, a partir de la tipología del papel empleado para la confección del documento, así como de la presencia y características de las filigranas y del hecho de que el uso de la foliación como elemento de ordenación en este tipo de documentos - aunque existen casos localizados en los siglos X-XI - se generaliza a partir del s. XVI, siendo muy escasos los ejemplares anteriores a esta data ${ }^{10}$, hemos de concluir que en ningún caso este manuscrito puede situarse en el siglo XII, datación que habrá de ser recogida con reservas en su catalogación a la espera de su confirmación por otros especialistas.

\section{El manuscrito ms 10}

El ms10 compila nueve obras bibliográficamente independientes que tratan temas relacionados con la filología árabe, la crítica y la interpretación ${ }^{11}$ en las que aparecen dos manos de escritura: la primera hasta el folio $278 \mathrm{v}$ y la segunda desde este mismo folio hasta el final del manuscrito. De ellas, según el catálogo, sólo tres hacen mención expresa a la data de copia. Son la segunda (ff. 6v-73r), Šarh al-qașìda al-Jazraŷiyya -1413 d.C.- , tercera (ff. 74v-129r), Šarh qașīda Diya ${ }^{\prime}$ al-Dīn al-Jazraŷ̀̃ -1704 d.C.- y cuarta obra (ff. 130r-263v), AlRāyāt al-samhariyya fi šarh al-maqșūra al-Jazraŷiyya -1705 d.C.-Para su manufactura se utilizaron dos tipos de papel de lino y filigranados diferentes: uno para los folios 1 a 129, donde se recogen las obras una, dos y tres, y otro, desde el 130 hasta el final, para el resto de los textos compilados. En el primero el espesor es $0,7 \mathrm{~mm}$ y en el segundo

${ }^{10}$ Ruiz García, Introducción a la codicología, p. 167.

${ }^{11}$ Gonzalez García, Estudio de las encuadernaciones originales, anexos, pp. 47-48. 
$0,11 \mathrm{~mm}$, sin variaciones que alteren la regularidad a lo largo de toda la hoja. El primero tiene siete corondeles por folio y el segundo ocho, con distancias bastante similares, con poca diferencia de grosor entre ellos mostrando, incluso, coincidencias en algún caso.

Tabla 1

Características físicas del papel en el ms10

\begin{tabular}{|c|c|c|c|c|c|c|}
\hline \multirow[b]{2}{*}{ Ref } & \multicolumn{3}{|c|}{ Corondeles } & \multirow{2}{*}{$\begin{array}{l}\text { Puntizones } \\
\mathrm{mm} / \mathbf{2 0} \mathrm{p}\end{array}$} & \multirow[b]{2}{*}{ Filigrana } & \multirow{2}{*}{$\begin{array}{c}\text { Espesor } \\
(\mathrm{mm})\end{array}$} \\
\hline & $\mathbf{N}^{0}$ & $\begin{array}{c}\text { Distancia entre } \\
\text { ellos }(\mathrm{mm})\end{array}$ & Disposición/lomo & & & \\
\hline Papel 1 & 7 & $20,26,31$ & Perpendicular & 21 & $\begin{array}{c}1+1 \\
\text { contramarca }\end{array}$ & 0,7 \\
\hline Papel 2 & 8 & $22-24$ & Perpendicular & 18 & 1 & 0,11 \\
\hline
\end{tabular}

En el estudio de la filigrana comprobamos que el primero presenta la filigrana del círculo con puño y contramarca letras TP con una flor, diseño situado cronológicamente en el s. XVIII, y el segundo, una campana con cartela y corona en la parte superior (figura 3) que se desarrolla en este mismo periodo.

Figura 3

Filigranas y contramarca del ms 10

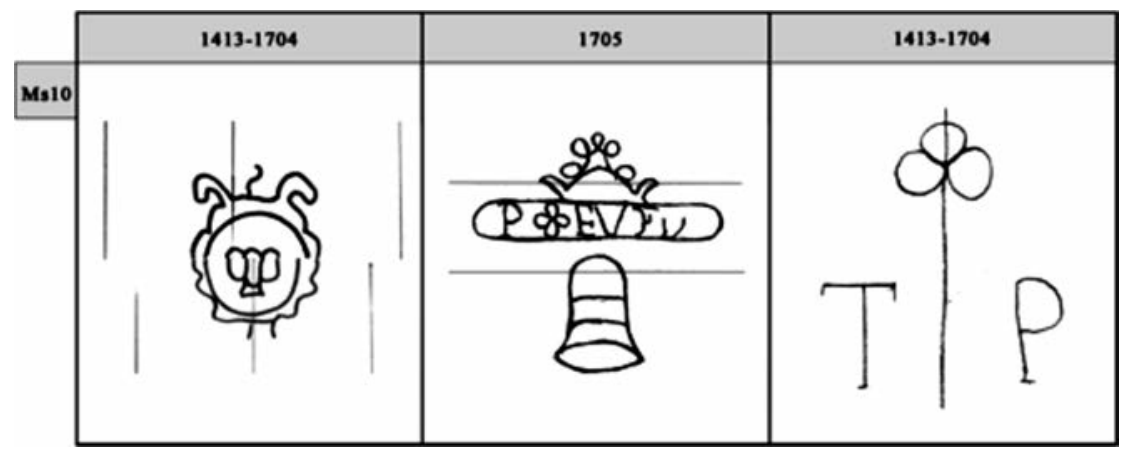


Sobre la autoría de la copia, el análisis del texto determina la presencia de dos manos de escritura, una desde el inicio del manuscrito hasta el folio $278 \mathrm{v}$ - obras 1 a 8 - y otra desde este mismo folio hasta el final, - obra 9-.

Estos datos evidencian una misma mano de ejecución del texto sobre los dos papeles utilizados y, dado que las filigranas los sitúan en el siglo XVIII, debemos descartar 1413 - fecha recogida en el colofón de la segunda obra - como data del manuscrito en favor de las que aparecen en las obras 3 y 4 . En consecuencia, se deduce que todo el ejemplar se debió copiar entre los años 1704 y 1705.

\section{El manuscrito ms26}

El ms26 incluye cinco obras cuya temática se centra en la filosofía islámica y la lógica. De ellas sólo la segunda, Tahrīr al-qawā'id almanțiqiyya fì šarh al-risāla al-šamsiyya, 一ff. 32v-101r - tiene data, en 1329 d.C. Todo el ejemplar utiliza un único tipo de papel ${ }^{12}$. Su espesor es regular aunque con una pequeña oscilación de $0,2 \mathrm{~mm}-0,12$ $0,14 \mathrm{~mm}$ - Se han observado siete corondeles por folio y mayor separación entre puntizones -20 puntizones ocupan $25 \mathrm{~mm}$ - que la habitual en un papel de manufactura italiana, recordando más el uso de formas vegetales.

Encontramos que la marca de agua repite el motivo de las tres lunas asociada a la VG; sólo en un caso se ha podido observar la relación entre ambos elementos. En el resto no se ha podido comprobar si coinciden en el mismo bifolio por imposibilidad técnica. Aun así, los indicios nos hacen pensar que también vayan asociados. Todas las referencias encontradas las sitúan en un periodo posterior a la fecha que presenta la segunda obra, pues la representación de las tres lunas fue un motivo muy repetido en papeles durante los siglos XVII y XVIII, continuando su producción hasta el s. XIX ${ }^{13}$.

\footnotetext{
${ }^{12}$ Entre los folios 104 y 107 el tono del papel es un poco más oscuro, aunque el estudio de la verjura, la filigrana y la fibra determinan que se trata del mismo papel que en el resto del volumen.

${ }^{13}$ Wiesmüller, "The Watermarks from the Refaiya Library".
} 
Figura 4

Filigrana y contramarca del ms26

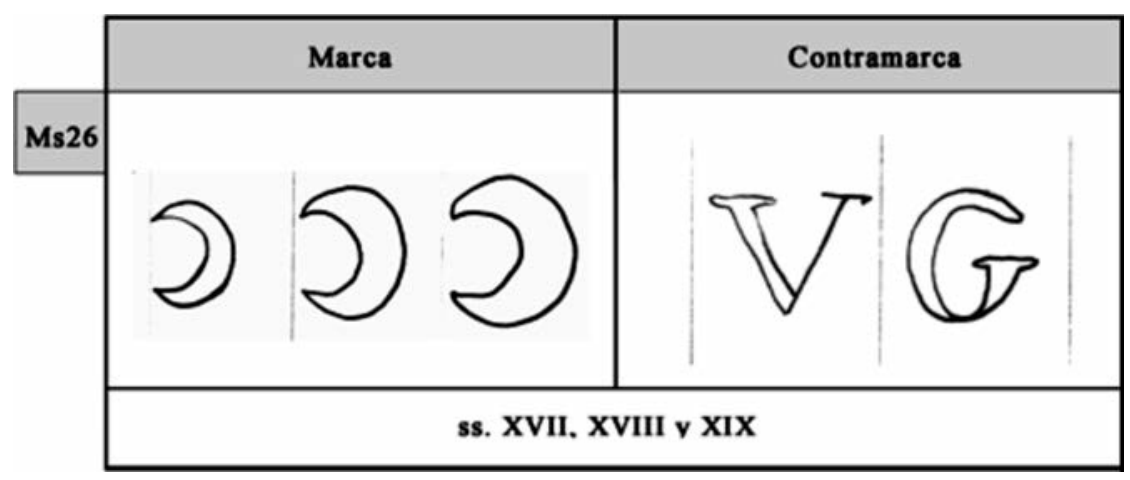

Otro dato relevante y decisivo para la datación del documento resulta de la identificación de azul ultramar artificial ${ }^{14}$, fabricado únicamentedesde 1829 a partir del lapislázuli (figura 6), como el pigmento utilizado para conformar la tinta azul utilizada para delinear la caja que enmarca el texto a lo largo de todo el manuscrito.

Figura 5

Izda: Macrofotografia de tinta azul folio 194r del ms26. Dcha: espectro obtenido mediante SEM/EDX de una muestra tomada de la tinta azul.
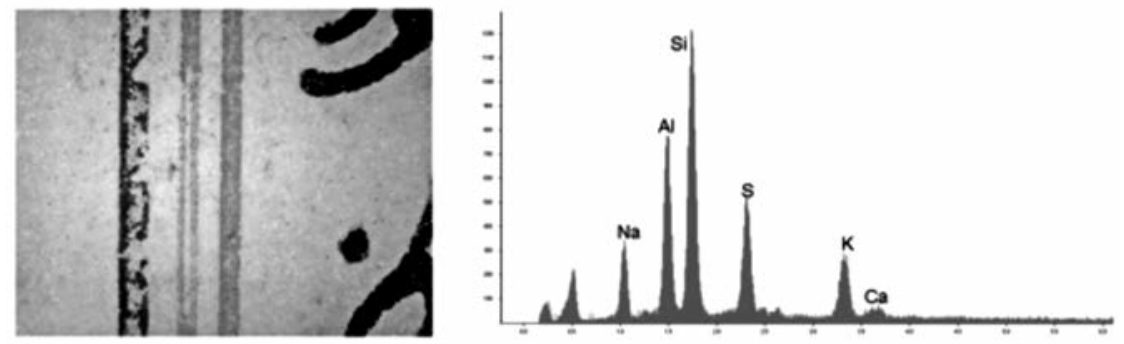

Igualmente, una mandorla central idéntica a la que se localiza en el ms37 — datado en el s. XIX — ha sido utilizada como elemento decorativo de la cubierta del ejemplar que nos ocupa.

${ }^{14}$ Plesters, "Ultramarine blue, artificial”, pp. 55-65 
Figura 6

Mandorlas centrales idénticas en ms26 y ms37.
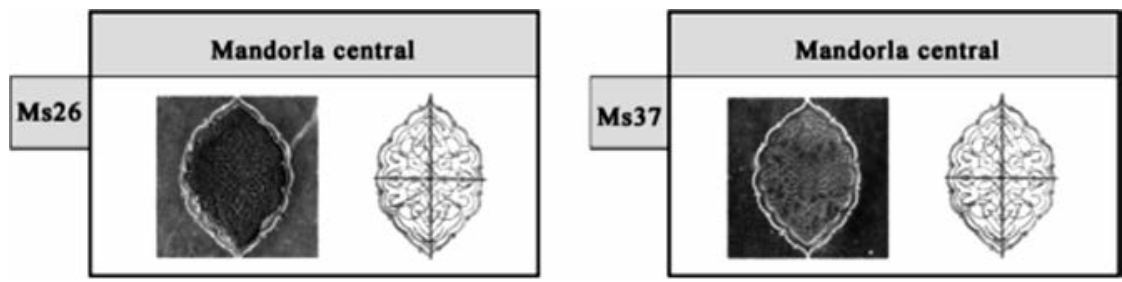

A todos estos datos hay que añadir que el manuscrito está copiado por la misma mano y que, con la salvedad de Muhammad b. Qutb al-Taḥtānī, autor de la segunda obra y coetáneo a la data expresada, el resto vivieron en los siglos XVII y XVIII —al-Jabīṣī, 'Ubayd Allāh b. Faụl Allāh (m. ca. 1640); Muḥammad b. Bannānī (1720 ó 21-1780) y Muhammad al-Ṭayyib b. Ibn Kīrān (1758 ó 9-1812 ó 13), a caballo entre los siglos XVIII y XIX ${ }^{15}$, por lo que en ningún caso, pudo ser en una fecha anterior a esta última.

En consecuencia la data expresada no puede corresponderse con la ejecución del manuscrito.

\section{El manuscrito ms29}

Este ejemplar presenta cinco obras cuyo tema común es el derecho islámico. De ellas, cuatro tienen data: la primera, Šarh Tuhfat alhukkām (ff. 5v-224v) - 1848? —, la segunda, Ta 'ī̄q waŷizz 'alà a-lāmiyya [sic] al-Zaqqāq (ff. 226r-292r) -1849?-, la cuarta, Al-Amāliyyāt al-fāšiyya fi šarḥ al-'Amāliyyāt al-fāsiyya (ff. 336r-430r) —1709-1710 — y la quinta, Bustān fikr al-minhaŷ fi tadlīl muntajab al-minhaŷ (ff. 430r-441v) entre 1649 y 1650.

El análisis físico y químico del papel empleado como soporte en los documentos compilados es coincidente, demostrando la utilización de

${ }^{15}$ Maŷmū'a mu'allafāt fì 'ilm al-manțiq wa-l-falsafa, en http://manuscripta.bibliotecas.csic.es

${ }^{16}$ El catálogo presenta una doble fecha: $1709-10$ en la ficha de la obra y 1848 en la ficha general del manuscrito. Hemos considerado la primera como válida porque se corresponde con la fecha que aparece en la transcripción del colofón: 1121/1709-10. 
un único tipo. Su espesor es de $0,9 \mathrm{~mm}$, muy regular en todos los folios, sin verjura ni filigrana y con una dirección de fibra preferente que aleja su producción de la elaboración en tina. Este hecho, unido al empleo de una única mano de escritura en todo el manuscrito, sitúan su ejecución, incluida la quinta obra, alrededor de la mitad del s. XIX.

\section{Conclusiones}

Los datos obtenidos de la aplicación del protocolo de caracterización para el estudio de los manuscritos - puestos en relación con aquellos derivados de la bibliografía y la experiencia del equipo investigador - demuestran la idoneidad del método para completar la información existente sobre cada uno de los ejemplares y corregir errores de descripción a la vez que nos acercan a la datación de los ejemplares y revelan la eficacia del análisis codicológico como requisito imprescindible para una correcta catalogación.

Con los resultados obtenidos se concluyó que al menos las datas expresadas en los mss 6, 10, 26 y 29 no se corresponden con el periodo de ejecución lo que obliga a revisar su catalogación, tal y como se detalla a continuación:

Frente a la data expresada para el ms6 - 1190 d.C.- el estudio del papel y los elementos de ordenación sitúan su momento de ejecución en fechas posteriores al siglo XVI.

En el ms 10 resulta obligado revisar la fecha que aparece en colofón - 1413 d.C - para situar el ejemplar en el siglo XVIII, concretamente entre 1704-1705.

En el ms26 ha resultado especialmente relevante el análisis material del color y el periodo de actuación de los autores para situar su data en el siglo XIX y no en 1329 d.C como expresaba una de las obras compiladas.

La relación entre las datas expresas de las obras reunidas en el ms 29 y las características de fabricación del papel han permitido localizar este documento a mediados del siglo XIX.

En este sentido hay que destacar que, debido a la diversidad de los elementos objeto de estudio, para llegar a estas conclusiones ha sido imprescindible la colaboración de un equipo multidisciplinar de trabajo capaz de abordar y poner en relación el análisis filológico, físico y analítico en todos sus aspectos. 


\section{Bibliografía}

Álvarez de Morales, Camilo y Orihuela Uzal, Antonio, La casa del Chapiz, Granada, CSIC - Universidad de Granada, 2013.

Ashok, Roy (ed.), Artist's Pigments, Washington/Nueva York/Oxford, National Gallery of Arts (Washington D.C.) and Oxford University Press, vol.2., 1997.

Ávila, María Luisa, Font, Miriam y de la Torre, Concha (coords.), Manuscritos árabes y fondo antiguo de la Escuela de Estudios Arabes: exposición con motivo del 75 Aniversario de la fundación de la Escuela de Estudios Arabes (CSIC), Granada, CSIC, 2007.

Basanta Campos, José Luis (coord.), Marcas de agua en documentos de los archivos de Galicia. Siglo XIX. Tomo VII y VIII, La Coruña, Fundación Pedro Barrié de la Maza, 2002.

Basanta Campos, José Luis (coord.), Marcas de agua en documentos de los archivos de Galicia. Siglo XVIII. Tomo Vy VI, La Coruña, Fundación Pedro Barrié de la Maza, 2000.

Basanta Campos, José Luis (coord.), Marcas de agua en documentos de los archivos de Galicia. Siglo XVII. Tomo III y IV, La Coruña, Fundación Pedro Barrié de la Maza, 1998.

Basanta Campos, José Luis (coord.), Marcas de agua en documentos de los archivos de Galicia hasta 1600. Tomos I y II, La Coruña, Fundación Pedro Barrié de la Maza, 1996.

Castilla Brazales, Juan, "La Escuela de Estudios Árabes en su 75 aniversario", Awrāq: Estudios sobre el mundo árabe e islámico contemporáneo, 23 (2006), pp. 289-298.

Castillo Castillo, Concepción, "Manuscritos arábigos que se conservan en la Escuela de Estudios Árabes de Granada", Cuadernos de Historia del Islam, 11 (1984), 213-238.

Espejo, Teresa y Beny, Ana, "Protocolo de descripción del manuscrito andalusí", en Teresa Espejo y Juan Pablo Arias (eds.), El Manuscrito Andalusí. Hacia una denominación de origen, Granada, Junta de Andalucía. Conserjería de Cultura, 2008, pp. 87-134.

González García, Sonsoles, Estudio de las encuadernaciones originales datadas de la Colección de manuscritos árabes de la Biblioteca de la Escuela de Estudios Árabes de Granada, Tesis Doctoral, Granada, Universidad de Granada, 2014.

Martínez de Sousa, José, Diccionario de bibliología y ciencias afines, Gijón, Editorial Trea, 2004.

Montalbán, Juan Antonio, "Del zigzag a la filigrana: Aproximación a una tipología del papel hispanoárabe", en VII Congreso Nacional de Historia del papel en España: Actas, Madrid, Asociación Hispánica de Historiadores del Papel, 2007, pp. 69-80. 
Plesters, Joyce, "Ultramarine blue, artificial", en Roy Ashok (ed.), Artist's pigments, 2 vols., Washington y Oxford, National Gallery of Arts, Oxford University Press, 1997, pp. 55-65

Ruiz García, Elisa, Introducción a la codicología, Madrid, Fundación Sánchez Ruizpérez, 2002.

Wiesmüller, Beate, "The Watermarks from the Refaiya Library", www.refaiya.unileipzig.de/texts/Watermarks_Refaiya.pdf. (11 febrero 2017).

Recibido: $12 / 09 / 2016$

Aceptado: 07/03/2017 